\title{
IV. Ueber den Azurit vom Laurion - Gebirge in Griechenland.
}

\author{
Von
}

K. Zimángi in Budapest*).

(Hierzu Taf. V, Fig. 20-23.)

In neuerer Zeit gelangten in die Sammlungen sehr schöne Exemplare des krystallisirten Azurites von Laurion.

Nachdem mir durch die Güte des Herrn Prof. J. A. K ren ner Gelegenheit geboten wurde, zu den Messungen besonders geeignete Krystalle zu untersuchen, will ich meine Ergebnisse im Folgenden kurz mittheilen.

Wie ich an den Handstucken beobachten konnte, kommen die Azuritkrystalle auf dem den Limonit durchsetzenden unreinen Cuprit (Ziegelerz) vor; das gewöhnliche Begleitmineral ist dünnfaseriger Malachit.

Die Krystalle sind durchschnittlich $0,5-8 \mathrm{~mm}$ lang, bei einer Breile von $0,5-2 \mathrm{~mm}$; zu den Messungen habe ich nur die kleinen ( $1-3 \mathrm{~mm}$ ) Individuen ausgewählt, da diese die glattesten Flächen besassen. Das aufgewachsene Ende der Krystalle ist nicht selten, wenigstens oberflachlich, in Malachit umgewandelt.

Der Azurit von Laurion reiht sich, was die Schönheit der Krystalle und Anzahl der Formen anbelangt, den interessantesten Vorkommen von Chessy**), Arizona ${ }^{* * *}$ ) und Utah ${ }^{*}$ ) an.

Die Krystalle weisen den häufigsten Typus dieses Minerals auf, indem dieselben gestreckt sind in der Richtung der Orthodiagonale; die Individuen

*) Aus dem ung. Original (Mathem. és természettud. Értesitö, 1892, 10, 198) vom Verf. mitgetbeilt. Vorgelegt der ung. Akademie in der Sitzung vom 16. Mai 1892 von Prof. J. A. Krenner. 2, 190.

**) Sitzungsber. d. Wiener Akad. 1871, 64, 123. D es Cloizeaux, Manuel, 1874,

***) Diese Zeitschr. 1887, 12, 319 und Americ. Journ. Sc. 1891, 41, 300.

f) Diese Zeitschr. 1884, 8, 532. 
sind beinahe ausschliesslich mit einem Ende der Symmetrieaxe aufgewachsen. Meistens herrschen zwei oder drei Orthodomen den übrigen vor, wodurch der flachprismatische Habitus (Fig. 20 und 22, Taf. V) entsteht; seltener sind die Dimensionen der Dicke in den zwei Richtungen nahezu gleich (Fig. 23).

Sieben Krystalle wurden ausführlich gemessen und die folgenden $\mathbf{2 8}$ Formen beobachtet, von denen die mit einem * bezeichneten neu sind. Die Symbole sind auf die Grundform Haidinger's*), Zippe's**) und Miller's***) bezogen, welche auch $\mathrm{D}$ a n a ${ }^{*}$ ) annahm.

$$
\begin{aligned}
& a=\{100\} \infty \pm \infty \\
& l=\{023\} \frac{2}{3} R \infty \\
& b=\{010\} \infty R \infty \\
& f=\{011\} R \infty \\
& c=\{001\} 0 P \\
& p=\{021\} 2 R \infty \\
& \sigma=\{101\}-P \infty \\
& m=\{110\} \infty P \\
& D=\{\bar{T} 04\} \frac{1}{4} P_{\infty} \\
& h=\{221\}-2 P \\
& F=\{\overline{2} 07\}\}_{7}^{2}+\infty \\
& s=\{111\}-P \\
& A=\{103\} \frac{1}{3} \mathbb{P}_{\infty} \\
& Q=\{223\}-\frac{2}{3} P \\
& { }^{*} I=\{\overline{2} 05\} \text { 䒺 } P \infty \\
& k=\{\overline{2} 21\} 2 P \\
& n=\{T 02\} \frac{1}{2} P \infty \\
& x=\{\text { T11 }\} \\
& { }^{*} T=\{\overline{4} 05\} \quad+\infty \infty \\
& e=\{\overline{2} 45\} \frac{4}{5} R 2 \\
& \boldsymbol{\theta}=\{\overline{1} 01\} \mathbf{P}_{\infty} \\
& d=\{\overline{2} 43\} \frac{4}{3} R 2 \\
& { }^{*} W=\{\overline{6} 05\} \frac{6}{5}+\infty \\
& o=\{\overline{2} 41\} 4 R 2 \\
& B=\{\overline{5} 04\} \frac{5}{4} P \infty \\
& \gamma=\{121\}-2 R 2 \\
& v=\left\{\overline{2}_{0} 1\right\} 2 P_{\infty} \\
& \varrho=\{\bar{T} 34\}_{\frac{3}{4}} R 3 \text {. }
\end{aligned}
$$

In den negativen Oktanten ist die Zabl der Formen nicht gross, hingegen sind die positiven Orthodomen am zahlreichsten vertreten.

Die Flächen der Hemidomenzone zeigen eine mehr oder weniger starke Streifung, bei grösserer Ausdehnung sind dieselben auch etwas gekrümmt. Die Basis $\{001\}$ und die Querflache $\{100\}$ haben eine glatte Beschaffenheit, oder ist an ihnen die Streifung nur schwach angedeutet; erstere Flaiche ist grösser entwickelt als letztere.

In der Orthodomenzone sind die häufigsten Formen $\{100\},\{001\},\{101\}$, $\{\overline{1} 03\},\{\bar{T} 01\},\{\overline{2} 01\}$.

Charakteristisch für dieses Vorkommen können einige Orthodomen angesehen werden, welche bisher selten oder nur einmal beobachtet wurden, es sind dies $\left\{T_{04}\right\},\{\overline{2} 07\},\left\{T_{03}\right\}$ und $\{\overline{5} 04\}$.

*) Fr. Mohs, Treatise on Mineralogy. Translated by W. Haidinger. 3 Vol. Edinburgh, 1825, 2, 167-171.

**) Pogg. Ann. 1831, 22, 393.

***) Philipp's Mineralogy by B rooke and Miller. London 1852, 594.

f) J. D. Dana, The System of Mineralogy. Sixth edition by E. S. D a na. New York 1892, 295. 
Die Form $\left\{\right.$ T03\} beobachlete bisher nur Schra u $\Upsilon^{*}$ ) an den Krystallen von Chessy; bei dem laurioner Azurit ist diese eine häufige Form, und zwar oft mit grossen Flächen (Fig. 20).

$\{T 01\}$ ist meislens vorherrschend unter den Orthodomen.

Die Flächen der neuen Orlhodomen waren sehr schmal und glatt, wenn breiter, dann zart gestreift. Auf diese mangelhafte Ausbildung ist das grössere Differiren der gemessenen und berechneten Winkelwerlhe zurückführbar. Die gestreiften Flächen gaben mehrere Reflexe; jedoch konnte ich zum Einstellen immer einen schwachen, aber scharfen und gui unterscheidbaren finden.

$I\{\overline{2} 05\}$ beobachlete ich an drei Krystallen mit je einer Fläche.

$$
\begin{array}{cc}
\text { Beobachtet: } & \text { Berechnet: } \\
(\overline{2} 05):(001)=21^{0} 42^{\prime}-22^{0} 34^{\prime} & 22^{\circ} 51^{\prime}
\end{array}
$$

$T\{\overline{4} 05\}$ wurde an zwei Krystallen constatirt, und zwar an einem mit voller Flächenzahl zur Entwickelung gelangt.

$$
\begin{array}{cc}
\text { Beobachtet : } & \text { Berechnet: } \\
(\overline{4} 05):(001)=4.0^{0} 19^{\prime}-40^{0} 24^{\prime} & 40^{0} 37^{\prime}
\end{array}
$$

Die Form $W\{\overline{6} 05\}$ beobachtete ich ebenfalls an zwei Krystallen.

$$
\begin{array}{cc}
\text { Beobachtet: } & \text { Berechnet: } \\
(\overline{6} 05):(001)=51^{0} 39^{\prime}-52^{0} 55^{\prime} & 52^{0} 39^{\prime}
\end{array}
$$

Nach diesen Messungen differiren die beobachteten Winkel beträchllich, ebenso ist die Abweichung von den theoretischen Werthen eine ziemlich grosse; die Signalbilder waren aber von genügender Schärfe, um die Messungsresultate zur Bestimmung der Symbole zu verwenden.

Das einzige negative Orthodoma $\{101\}$ war an jedem der untersuchten Krystalle mit schmalen Flächen entwickelt.

Auffallend schön ist die glatte Beschaffenheit der terminalen Flächen; wenn dennoch einzelne Messungen grössere Abweichungen von den berechneten Neigungen zeigen, so ist das nur Folge der lichtschwachen Reflexe der kleinen Flächen.

Von diesen Formen sind $\{110\},\{021\},\{221\},\{111\},\{223\},\{\overline{2} 41\},\{\overline{2} 43\}$ die häufigsten.

Mit Ausnahme von $\{\bar{T} 34\}$ gehören alle Pyramiden zu der Zone [110:001] oder zu [120:001].

Unter den Pyramiden der Hauptreihe ist $\{223\}$ die bestentwickelle; es ist das die seltene Form, welche zuerst $\mathrm{K}$ renner und Franzen a $\mathrm{u}^{* *}$ ) an

*) Sitz.-Ber. d. Wiener Akad. 1871, 64, 138.

**) Diese Zeitschr. 1884, 8, 532. 
den Krystallen von Utah erkannten und unlängst farringto $\mathbf{n}^{*}$ ) an dem Vorkommen von Arizona ebenfalls constatirte.

Betreffend das Grössenverhältniss der terminalen Flächen kann ich bemerken, dass $\{021\},\{110\},\{221\},\left\{\overline{2}_{41}\right\}$ gewöhnlich mit ausgedehnten Flächen auftreten; dahingegen sind schon kleiner $\{223\},\{\overline{\mathbf{2}} 43\}$.

Die zwei Formen $\{121\}$ und $\{134\}$ habe ich nur an der flächenreichsten Combination (Fig. 22) mit je einer, sehr untergeordneten Fläche beobachlet; ihre Lage wurden durch approximative Messungen und die Zonen bestimmt. Die erstere erkannte ich als schmale Abstumpfung der Kante [110:011] in den Zonen [001:24T] und [T01:110]; das Zeichen der Klinopyramide $\{\bar{T} 34\}$ ist gegeben durch die Zonen [023: T11] und [T01:011].

Die Klinodomen $\{023\}$ und $\{011\}$ habe ich ebenfalls nur an der complicirtesten Combination mit voller Flächenzahl an dem freien Ende des Krystalles beobachtet.

Die Symmetrieebene erkannte ich nur einmal als sehr schmale Abstumpfung der Kante des Klinodomas $\{021\}$.

Besonders bemerkenswerth und interessant sind die Zonenverhältuisse, welche derartige waren, dass ich die Symbole der Endflächen theilweise aus denselben ohne Winkelmessungen bestimmen konnte.

Sämmtliche von diesem Fundorte von mir beobachteten Formen habe ich in der Projection Fig. 21 eingetragen und die wichtigsten Zonenkreise gezeichnet.

In den drei Krystallbildern (Fig. 20, 22, 23) ist das relative Grössenverhältniss möglichst berücksichtigt worden.

Aus der folgenden Zusammenstellung ist dic Combination der untersuchten Krystalle ersichtlich. Das freie Ende des Krystalles Nr. 7 war abgebrochen.

\begin{tabular}{c|c|c|c|c|c|c|c}
\hline & \multicolumn{7}{|c}{ Nummer des Krystalles } \\
\cline { 2 - 7 } Formen & 1. & 2. & 3. & 4. & 5. & 6. & 7. \\
\hline$a\{100\}$ & $\times$ & $\times$ & $\times$ & $\times$ & $\times$ & $\times$ & $\times$ \\
$b\{010\}$ & & $\times$ & & & & & \\
$\boldsymbol{c}\{001\}$ & $\times$ & $\times$ & $\times$ & $\times$ & $\times$ & $\times$ & $\times$ \\
$\sigma\{101\}$ & $\times$ & $\times$ & $\times$ & $\times$ & $\times$ & $\times$ & $\times$ \\
$D\{104\}$ & & & $\times$ & & $\times$ & & \\
$F\{207\}$ & & & & & $\times$ & $\times$ & $\times$ \\
$A\{103\}$ & $\times$ & $\times$ & & $\times$ & $\times$ & $\times$ & $\times$ \\
$I\{\overline{2} 05\}$ & & $\times$ & $\times$ & & $\times$ & & \\
$n\{\overline{1} 02\}$ & $\times$ & $\times$ & $\times$ & & & & $\times$ \\
$T\{\overline{4} 05\}$ & & & & & $\times$ & $\times$ & \\
$\Theta\{101\}$ & $\times$ & $\times$ & $\times$ & $\times$ & & $\times$ &
\end{tabular}

*) On crystallized Azurit from Arizona. Americ. Journ. Sc. 1891, 41, 300. 


\begin{tabular}{|c|c|c|c|c|c|c|c|}
\hline \multirow{2}{*}{ Formen } & \multicolumn{7}{|c|}{ Nummer des Krystalles } \\
\hline & 1. & 2. & 3. & 4. & 5. & 6. & 7. \\
\hline$W\{\overline{6} 05\}$ & & $x$ & & & $x$ & & \\
\hline$B\{\overline{5} 04\}$ & & & & & $x$ & & \\
\hline$v\{\overline{2} 01\}$ & $x$ & $x$ & $x$ & $x$ & $x$ & $x$ & \\
\hline$l\{023\}$ & $x$ & & & & & & \\
\hline$f\{011\}$ & $x$ & & & & & & \\
\hline$p\{021\}$ & $x$ & $x$ & $x$ & $x$ & & $x$ & \\
\hline$m\{110\}$ & $x$ & $x$ & $x$ & $x$ & $x$ & & \\
\hline$h\{221\}$ & $x$ & $x$ & $x$ & $x$ & $x$ & $x$ & \\
\hline$s\{111\}$ & $x$ & $x$ & $x$ & $x$ & $x$ & & \\
\hline$Q\{223\}$ & $x$ & $x$ & $x$ & $x$ & $x$ & $x$ & \\
\hline$k\{\overline{2} 21\}$ & $x$ & & & & & & \\
\hline$x\{\bar{T} 11\}$ & $x$ & & & & $\times$ & & \\
\hline$e\{\overline{2} 45\}$ & $x$ & $\times$ & $x$ & & & & \\
\hline$d\{\overline{2} 43\}$ & $x$ & $x$ & $x$ & & $x$ & $x$ & \\
\hline$o\{241\}$ & $x$ & $x$ & $x$ & $x$ & $x$ & $x$ & \\
\hline$\gamma\{121\}$ & $x$ & & & & & & \\
\hline$\varrho\{134\}$ & $x$ & & & & & & \\
\hline
\end{tabular}

Die beobachteten und berechneten Neigungen sind in der nachstehenden Tabelle angeführt. Die berechneten Winkel leitete ich aus den Sch rauf'schen*) Elementen ab.

Beobachtet: Berechnet:

$$
\begin{aligned}
& c: a=(001):(100)=87^{\circ} 38^{\prime} \quad 8^{\prime} \quad 36^{\prime} \quad 0^{\prime \prime} \\
& : \sigma=\quad:(101)=4437 \quad 44 \quad 4556 \\
& : D=\quad:(\bar{T} 04)=1445 \text { ca. } \quad 143928 \\
& : F=\quad:(\overline{2} 07)=16 \quad 48 \quad 16 \quad 40 \quad 2 \\
& : A=\quad:(103)=19 \quad 6 \quad 19 \quad 1729 \\
& : I=\quad:(\overline{2} 05)=22 \quad 8 \quad 2250 \quad 37 \\
& : n=:(102)=2750 \quad 275229 \\
& : T=:(\overline{405})=4022 \quad 4037 \quad 6 \\
& : \Theta=:(101)=47 \quad 19 \quad 47 \quad 15 \quad 0 \\
& : W=\quad:(\overline{6} 05)=52 \quad 17 \quad 52 \quad 38 \quad 40 \\
& : B=\quad:(\overline{5} 04)=54 \quad 3 \quad 53 \quad 49 \quad 42 \\
& : v=\quad:(\overline{2} 01)=66 \quad 9 \quad 66 \quad 11 \quad 28 \\
& : l=\quad:(023)=\begin{array}{lll}
30 \quad 29 & 3023 \quad 32
\end{array} \\
& : f=\quad:(011)=4126 \quad 412050
\end{aligned}
$$

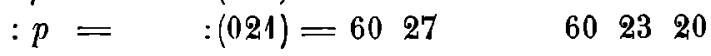

$$
\begin{aligned}
& : b=:(010)=9023 \mathrm{ca} . \quad 90 \quad 0 \quad 0 \\
& : Q=:(223)=41 \quad 20 \quad 41 \quad 21 \quad 2
\end{aligned}
$$

*) Sitzungsber. d. Wiener Akad. 1871, 64, 134. 


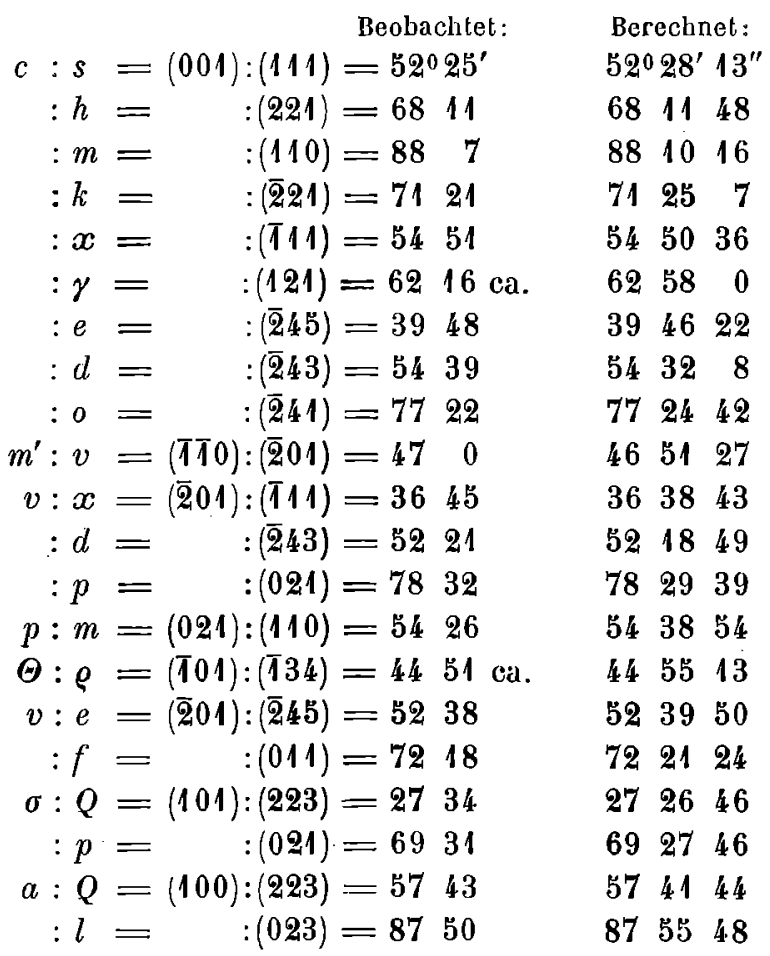

Im "Index der Krystallformen " giebt V. Goldschmidt*) 62 verschiedene Formen der Kupferlasur an; rechnet man hierzu die von Farring to ${ }^{* *}$ ) beobachteten drei neuen Pyramiden $\{\overline{4} 47\},\{\overline{3} 21\},\{\overline{1} \mathbf{2} .10 .5\}$, und die drei an dem Azurit von Laurion erkannten Orthodomen, so wären nunmehr an diesem Mineral zusammen 68 Formen constatirt.

Was die Anzabl der verschiedenen Formen und Combinalionen anbelangt, so werden die laurioner Krystalle nur von denjenigen von Chessy ubertroffen.

Es möge mir gestattet sein dem Herrn Prof. J. A. Krenner, Cuslos der mineralogischen Sammlung des ungarischen National-Museums, meinen besten Dank für die Gefälligkeit auszudrücken, mit welcher er mir aus der oben erwähnten Sammlung das schöne Material zur Untersuchung überliess.

Budapest, 1892, mineralogisches Institut des Polytechnikums.

*) Dr. Victor Goldschmidt, Index der Krystallformen der Mineralien. 3 Bde. Berlin 1886-1891, 2, 269-274.

**) Americ. Journ. Sc. 1891, 41, 300 